\title{
KÄHLER YAMABE MINIMIZERS ON MINIMAL RULED SURFACES
}

\author{
CHRISTINA W. TØNNESEN-FRIEDMAN
}

\begin{abstract}
It is shown that if a minimal ruled surface $\mathrm{P}(E) \rightarrow \Sigma$ admits a Kähler Yamabe minimizer, then this metric is generalized Kähler-Einstein and the holomorphic vector bundle $E$ is quasi-stable.
\end{abstract}

\section{Introduction}

The minimal ruled surfaces form a special class of compact Kählerian surfaces and are by definition the total spaces of $C P_{1}$ bundles over compact Riemann surfaces $\Sigma$. Any ruled surface can be written [2] as

$$
\mathrm{P}(E) \rightarrow \Sigma,
$$

i.e., as the projectivization of a holomorphic rank two vector bundle $E$ over $\Sigma$, where $E$ is unique up to tensoring with a holomorphic line bundle. Moreover any ruled surface is birationally equivalent to $\Sigma \times C P_{1}$. In particular, any ruled surface is algebraic. In fact, the minimal models of any complex surface which is birationally equivalent to $\Sigma \times C P_{1}$, are exactly the ruled surfaces [4], [22].

Suppose that $E \rightarrow \Sigma$ is quasi-stable, that is, $E$ is semi-stable (in the sense of Mumford) and decomposes into a direct sum

$$
E=E_{1} \oplus \cdots \oplus E_{k}
$$

of stable sub-bundles (here $k=1$ or 2) such that

$$
\frac{\operatorname{deg}(E)}{\operatorname{rank}(E)}=\frac{\operatorname{deg}\left(E_{i}\right)}{\operatorname{rank}\left(E_{i}\right)}
$$

for $i=1, \ldots, k$. Narasimhan and Seshadri [17] have proved that quasistability is equivalent to the existence of a flat projective unitary connection on $E$. In other words, if $E$ is quasi-stable, then $\mathrm{P}(E) \rightarrow \Sigma$ is a flat $\mathrm{C} P_{1}$ bundle, i.e., is defined by some representation

$$
\rho: \pi_{1}(\Sigma) \rightarrow \operatorname{PSU}(2)=\mathrm{SO}(3) .
$$

Received February 20, 1999. 
So, when $E$ is quasi-stable, local products of constant scalar curvature Kähler metrics on $\Sigma$ and $C P_{1}$ will exhaust the entire Kähler cone on the ruled surface with Kähler classes of constant scalar curvature Kähler metrics.

Burns and de Bartolomeis proved that quasi-stability is a necessary condition for the existence of scalar-flat Kähler metrics. More recently LeBrun proved a similar statement for negative constant scalar curvature. We summarize these results in the theorem below.

TheOREM 1.1 (Burns, de Bartolomeis [3] and LeBrun [12]). Let $\mathrm{P}(E) \rightarrow \Sigma$ be a minimal ruled surface with a Kähler class $[\omega]$ such that $c_{1} \cdot[\omega] \leq 0$. Then $[\omega]$ contains a Kähler metric of constant scalar curvature if and only if $E \rightarrow \Sigma$ is a quasi-stable vector bundle.

A key step in both proofs is the observation that the constant scalar curvature Kähler metric must be Kähler with respect to two non-equivalent complex structures on the ruled surface.

Whether the statement holds in the case $c_{1} \cdot[\omega]>0$ is still unknown. In this paper we assume that the Kähler metric is also a Yamabe minimizer in its conformal class and show that then quasi-stability holds.

\section{Perturbed Seiberg-Witten Invariants}

Let $M$ be a compact, oriented four manifold such that $H^{2}(M, \mathrm{R})$ has dimension two and $b_{+}=b_{-}=1$. (In general, one could let $b_{-}$have arbitrary value.) Let $g$ be a Riemannian metric on $M$ and $\star$ be the Hodge Star operator defined with respect to $g$ and the orientation. Then the one dimensional subspace of $H^{2}(M, \mathrm{R})$

$$
H^{+}(g):=\left\{[v] \in H^{2}(M, \mathrm{R}) \mid \star v=v\right\}
$$

is called a metric polarization [12]. Observe that

$$
H^{-}(g):=\left\{[v] \in H^{2}(M, \mathrm{R}) \mid \star v=-v\right\}
$$

is the metric polarization with respect to the opposite orientation. If $g$ is Kähler, then the Kähler class spans $H^{+}(g)$.

The open cone

$$
\left\{[v] \in H^{2}(M, \mathrm{R}) \mid[v] \cdot[v]>0\right\}
$$

consists of two connected components, called nappes [13]. Given a nappe $\mathscr{C}^{+}$ and a Riemannian metric $g$, let $\omega$ be a $g$-harmonic, self-dual two form such that $[\omega] \in \mathscr{C}^{+}$. This form always exists and is unique up to multiplication with a positive constant. Indeed, $[\omega] \in H^{+}(g) \cap \mathscr{C}^{+}$. If $M$ has a Kähler metric, then the canonical choice of nappe is the one containing the Kähler class. This 
way the corresponding $g$-harmonic, self-dual two-form to any metric on $M$ is simply a generalization of the Kähler form.

Now assume that $M$ has a $\operatorname{Spin}_{c}$ structure $c$ of almost-complex type. Relative to any metric $g$, the perturbed Seiberg-Witten invariant $p_{c}\left(M, \mathscr{C}^{+}\right)$is defined to be the number of solutions, modulo gauge and counted with orientations, of the perturbed Seiberg-Witten equations [10], [21]

$$
\begin{aligned}
D_{A} \Phi & =0 \\
i F_{A}^{+}+\sigma(\Phi) & =\epsilon,
\end{aligned}
$$

where $\epsilon$ is a generic (so that $(g, \epsilon)$ is excellent) self-dual two form with $\int_{M} \epsilon \wedge$ $\omega>2 \pi c_{1}(c) \cdot[\omega]$. Note that all $\epsilon$ satisfying this inequality make $(g, \epsilon)$ a good pair and it is easy to see that they are all in the same chamber. The above invariant is therefore well-defined and metric independent. We refer to [11] for definitions of the words "excellent", "good" and "chamber".

Note that if $p_{c}\left(M, \mathscr{C}^{+}\right) \neq 0$ the the equations (1) and (2) have a solution $\Phi \neq 0$ for any $\epsilon=t \omega$ where $t \gg 0$. This is easily seen by the fact that $(g, t \omega)$ is a good pair (in the chamber determined by $\mathscr{C}^{+}$). If it had no solutions, it would automatically be excellent and therefore contradict the non-vanishing of $p_{c}(M, \mathscr{C})$. Therefore, $(g, t \omega)$ has a solution (not necessarily transverse) and by $(g, t \omega)$ being good this solution is irreducible $(\Phi \neq 0)$.

ExAmPLE 2.1 ([11]). If $(M, J, g)$ is a Kähler surface, $c$ the $\operatorname{Spin}_{c}$ structure induced by $J$ and $\mathscr{C}^{+}$the canonical choice of nappe then $p_{c}\left(M, \mathscr{C}^{+}\right) \neq 0$.

The perturbed Seiberg-Witten invariant is also defined for $\operatorname{Spin}_{c}$ structures on $M$ who do not arise from an almost-complex structure [15].

If $(M, g, J)$ is an almost-Kähler manifold, then the almost-Kähler form $\omega$ is a harmonic self-dual form. Hence, even though $J$ may not be integrable, $[\omega]$ still determines a canonical choice of nappe. Since $|\omega|=\sqrt{2}$, the following result is a direct application of ([13], Theorems 1 and 2).

TheOREM 2.2 (LeBrun). Let $(M, g, J)$ be an almost-Kähler surface with the canonical choice of nappe $\mathscr{C}^{+}$. If c is a $\operatorname{Spin}_{c}$ structure such that $p_{c}\left(\mathscr{C}^{+}, M\right) \neq$ 0 , then

$$
\int_{M} s d \mu \leq 4 \pi c_{1}(c) \cdot[\omega]
$$

where $s$ is the scalar curvature, $d \mu$ is the metric volume form and $c_{1}(c)=$ $c_{1}\left(\operatorname{det} V^{+}\right)$. Moreover, equality is achieved if and only if $(M, g, J)$ is Kähler and $J$ is compatible with $c$.

For the proof we refer to LeBrun's paper [13]. However in ([13], Theorem 2) the compability statement was made without offering a proof. For the sake of 
completeness we now prove this. When equality is achieved, we have that $(M, g, J)$ is Kähler. Therefore

$$
4 \pi c_{1}(c) \cdot[\omega]=\int_{M} s d \mu=4 \pi c_{1}\left(K^{-1}\right) \cdot[\omega],
$$

where $K^{-1}$ is the anti-canonical line bundle of $(M, J)$. The compability of $J$ with $c$ then follows from the following lemma.

Lemma 2.3. Let $M$ be a compact smooth manifold with $b_{+}=1$. Assume that $M$ has a Kähler metric $g$ with Kähler form $\omega$ and complex structure J. Let $K$ denote the canonical line bundle of $(M, J)$. Let $\mathscr{C}^{+}$be the canonical choice of nappe. Suppose $c$ is any $\operatorname{Spin}_{c}$ structure on $M$ with corresponding complex line bundle $L=\operatorname{det} V^{+}$such that $p_{c}\left(M, \mathscr{C}^{+}\right) \neq 0$. Then $E=(K \otimes L)^{\frac{1}{2}}$ is either trivial or a holomorphic line bundle corresponding to an effective divisor. In particular, $c_{1}(L) \cdot[\omega] \geq c_{1}\left(K^{-1}\right) \cdot[\omega]$ with equality if and only if $E$ is trivial and $c$ is the $\operatorname{Spin}_{c}$ structure induced by $J$.

Proof. The trick is to choose the perturbation to be $\epsilon=t \omega, t \gg 0$. Now we follow Witten's calculations for the unperturbed Seiberg-Witten equations on a Kähler manifold [21] (see also the proof of ([8], Proposition 2.1)). Since $\epsilon$ is of type $(1,1)$ with respect to the Kähler structure, we get by precisely the same argument as in [21] that for a solution $(A, \Phi)$ to both equation (1) and

$$
i F_{A}^{+}+\sigma(\Phi)=t \omega
$$

the curvature $F_{A}$ is of type $(1,1)$ and $E$ has a holomorphic structure (induced by $\left.D_{A}\right)$. If we write $\Phi=(\alpha, \beta)$ where $\alpha$ is a section of $E$ and $\beta$ is a section of $\Lambda^{0,2}(E)$, then $\alpha$ and $\bar{\beta}$ are holomorphic and one of them must vanish. Now (3) rewrites to

$$
i F_{A}^{+}=\frac{\left(-|\alpha|^{2}+|\beta|^{2}+4 t\right)}{4} \omega
$$

implying that

$$
2 \pi c_{1}(L) \cdot[\omega]=\frac{\left(-|\alpha|^{2}+|\beta|^{2}+4 t\right)}{4}[\omega]^{2} .
$$

For $t$ sufficiently large we must have that $\alpha$ is a non-vanishing holomorphic section of $E$. Thus, unless it is trivial, the line bundle $E$ corresponds to an effective divisor. The inequality now follows from the fact that the "area" of any effective divisor on the Kähler manifold is non-zero.

If $E$ is trivial, then $L=K^{-1}$, and since a $\operatorname{Spin}_{c}$ structure on an almostcomplex manifold is determined by the determinant line bundle $L=\operatorname{det} V^{+}$, we are done. 
The author would like to point out that Lemma 2.3 is a special case of Theorem 1.3 in [19] where Taubes proved a similar statement in the symplectic setting.

\section{The Yamabe Constant}

Definition 3.1. Let $g$ be a Riemannian metric on a four manifold $M$. The Yamabe constant of the corresponding conformal class $[g]$ is defined to be

$$
Y_{[g]}=\inf _{g \in[g]} \frac{\int_{M} s_{g} d \mu_{g}}{\left(\int_{M} d \mu_{g}\right)^{\frac{1}{2}}} .
$$

Note that the above infimum is in fact achieved by a metric in $[g]$. This was proved by Yamabe, Trudinger, Aubin and Schoen [1], [14], [18]. A metric which minimizes $\left(\int_{M} s_{g} d \mu_{g}\right) /\left(\int_{M} d \mu_{g}\right)^{\frac{1}{2}}$ on $g$ is called a Yamabe minimizer. Any Yamabe minimizer must have constant scalar curvature. If $Y_{[g]} \leq 0$, then $g$ is the unique (up to scalar multiplication) Yamabe minimizer of $[g]$ if and only if $g$ has constant scalar curvature. Unfortunately, for $Y_{[g]}>0$, constant scalar curvature does not necessarily imply that a metric is a minimizer, and uniqueness of the minimizers does not always hold in this situation either. Observe that $Y_{[g]}>0$ if and only if there exists a metric in $[g]$ with strictly positive scalar curvature.

By applying Theorem 2.2, LeBrun found an estimate for $Y_{[g]}$.

Theorem 3.2 (LeBrun [13]). Let $(M,[g])$ be an oriented conformal Riemannian four-manifold, and let $\omega$ be a closed 2-form which is self-dual with respect to $[g]$ and not identically zero. Suppose that $b^{+}(M)=1$ and that the perturbed Seiberg-Witten invariant $p_{c}\left(M, \mathscr{C}^{+}\right)$is non-zero for some $\operatorname{Spin}_{c}$ structure $c$, where $\mathscr{C}^{+} \subset H^{2}(M, \mathrm{R})$ is the nappe containing $[\omega]$. Then the Yamabe constant of $[g]$ satisfies

$$
Y_{[g]} \leq \frac{4 \pi c_{1}(c) \cdot[\omega]}{\sqrt{[\omega]^{2} / 2}} .
$$

Moreover, equality is achieved if and only if there is a Yamabe minimizer $g \in[g]$ which is Kähler, with Kähler form $\omega$ and complex structure compatible with c.

Definition 3.3 ([16]). A Kähler metric is said to be generalized KählerEinstein if the Ricci form is parallel with respect to the Levi-Civita connection.

We can now prove the following theorem.

Theorem 3.4. Let $M=\mathrm{P}(E) \rightarrow \Sigma$ be a minimal ruled surface over a compact Riemann surface $\Sigma$. If $M$ has a Kähler metric $g$ with constant 
positive scalar curvature such that $g$ is a Yamabe minimizer in $[g]$, then $g$ is generalized Kähler-Einstein and therefore locally a product. Consequently $E$ is a quasi-stable holomorphic vector bundle.

If $g$ has constant non-positive scalar curvature, then the above is true by Theorem 1.1.

Proof. First assume that $\Sigma$ is $C P_{1}$. The only Hirzebruch surface with constant scalar curvature Kähler metric is the product $\mathrm{C} P_{1} \times \mathrm{C} P_{1}$ [6]. On this surface any constant scalar curvature Kähler metric must be invariant under the $\mathrm{SO}$ (3) action on each $\mathrm{C} P_{1}$. This forces the metric to be a product of (multiple of) the Fubini-Study metric. We have used the fact that any extremal Kähler metric is invariant under the action of the maximal compact subgroup of (the identity component of) the group of holomorphic transformations[7].

Now assume that the genus $\mathbf{g}$ of $\Sigma$ is at least one. Let $g$ be a Kähler Yamabe minimizer with positive scalar curvature. The Yamabe constant of $[g]$ is then given by

$$
Y_{[g]}=\frac{4 \pi c_{1} \cdot[\omega]}{\sqrt{[\omega]^{2} / 2}},
$$

where $\omega$ is the Kähler form of $g$ and $c_{1}=c_{1}\left(K^{-1}\right)$. Note that for a minimal ruled surface $b_{+}=b_{-}=1$. Let $c$ be the $\operatorname{Spin}_{c}$ structure induced by the complex structure $J$ on $M$ and let $\mathscr{C}^{+}$be the canonical choice of nappe. According to Example 2.1, $p_{c}\left(M, \mathscr{C}^{+}\right) \neq 0$ and we see that equation (4) is a special case of Theorem 3.2.

Now consider the fiber-wise anti-podal map $\psi: M \rightarrow M$ [12]. This is an orientation reversing diffeomorphism and we can define a $\operatorname{Spin}_{c}$ structure $\bar{c}$ on $\bar{M}$ by setting $\bar{c}:=\psi^{*} c$. That is, $\bar{c}$ is the canonical $\operatorname{Spin}_{c} \operatorname{structure}$ associated to $\psi^{*} J$. Observe that $\psi^{*}$ sends $\mathscr{C}^{+}$to a nappe $\psi^{*} \mathscr{C}^{+}$for $\bar{M}$ and $\psi^{*}\left(H^{+}(g)\right)=$ $H^{-}(g)$ [12]. Since $\left(\bar{M}, \bar{c}, \psi^{*} \mathscr{C}^{+}\right)$and $\left(M, c, \mathscr{C}^{+}\right)$are isomorphic as oriented four-manifolds with nappes and $\operatorname{Spin}_{c}$ structures, we have that

$$
p_{\bar{c}}\left(\bar{M}, \psi^{*} \mathscr{C}^{+}\right)=p_{c}\left(M, \mathscr{C}^{+}\right) \neq 0 .
$$

Theorem 3.2 applied to the original conformal class $[g]$ and $\left(\bar{M}, \bar{c}, \psi^{*} \mathscr{C}^{+}\right)$ now implies that

$$
Y_{[g]} \leq \frac{4 \pi \psi^{*} c_{1} \cdot \psi^{*}[\omega]}{\sqrt{\left(\psi^{*}[\omega]\right)^{2} / 2}}
$$

on $\bar{M}$. But the Yamabe constant is independent of orientation and the right hand side of (5) is just the right hand side of (4). So we must have equality in (5) and thus there exists a Yamabe minimizer $\tilde{g} \in[g]$ such that $\tilde{g}$ is Kähler 
with respect to some complex structure $\tilde{J}$ in $\bar{c}$, where the Kähler form $\tilde{\omega}$ is equal to the harmonic part of $\psi^{*} \omega$.

Now we want to show that $\tilde{g}=g$. We can assume that $\int d \mu=\int d \tilde{\mu}=1$. If we write $\tilde{g}=u^{2} g$ for some positive smooth function $u$, we have that

$$
\int u^{4} d \mu=1
$$

and

$$
|\tilde{\omega}|^{2}=\left.u^{4} \tilde{\mid} \tilde{\omega}\right|^{2}=2 u^{4} .
$$

Since $\tilde{s}=Y_{[g]}=s$, we have that

$$
\Delta u=\frac{s\left(u^{3}-u\right)}{6} .
$$

Since the Euler characteristic of $M$ is given by $\chi=4(1-\mathbf{g})$ and the signature $\sigma=b_{+}-b_{-}$vanishes, the (strict) Hitchin-Thorpe inequality [9], [20], [5], $2 \chi>3|\sigma|$, is not satisfied when $\mathbf{g}>0$. Therefore no Riemannian metric on $M$ can be Einstein. In particular, the primitive part $\rho_{0}$ (resp. $\tilde{\rho}_{0}$ ) of the Ricci form $\rho$ (resp. $\tilde{\rho}$ ) of $g$ (resp. $\tilde{g}$ ) does not vanish. Moreover, $d \rho_{0}=$ $d \tilde{\rho_{0}}=0$, which follows from the fact that the scalar curvatures are constant. Since $b_{+}=b_{-}=1$, we must therefore have that $\tilde{\omega}=k \rho_{0}$ and $\omega=\tilde{k} \tilde{\rho_{0}}$, where $k$ and $\tilde{k}$ are non-zero constants. Now $\psi^{*}\left[\frac{\rho}{2 \pi}\right]=\psi^{*} c_{1}=c_{1}(\bar{c})=\left[\frac{\tilde{\rho}}{2 \pi}\right]$. In particular,

$$
\psi^{*}\left[\rho_{0}\right]=\left[\tilde{\rho_{0}}\right],
$$

thus

$$
k^{-1} \psi^{*}[\tilde{\omega}]=\tilde{k}^{-1}[\omega]
$$

Hence

$$
k^{-1} \psi^{*} \psi^{*}[\omega]=k^{-1}[\omega]=\tilde{k}^{-1}[\omega]
$$

and consequently $k=\tilde{k}$.

We can calculate $k$ up to a sign as follows:

$$
\begin{aligned}
c_{1}^{2} & =\frac{1}{(2 \pi)^{2}} \int \rho \wedge \rho=\frac{1}{(2 \pi)^{2}}\left(\int\left(\frac{s}{4} \omega\right) \wedge\left(\frac{s}{4} \omega\right)+\int \rho_{0} \wedge \rho_{0}\right) \\
& =\frac{1}{(2 \pi)^{2}}\left(\frac{s^{2}}{8}-\int\left|\rho_{0}\right|^{2} d \mu\right)=\frac{1}{(2 \pi)^{2}}\left(\frac{s^{2}}{8}-k^{-2} \int|\tilde{\omega}|^{2} d \mu\right) \\
& =\frac{1}{(2 \pi)^{2}}\left(\frac{s^{2}}{8}-2 k^{-2} \int u^{4} d \mu\right)=\frac{1}{(2 \pi)^{2}}\left(\frac{s^{2}}{8}-2 k^{-2}\right),
\end{aligned}
$$


and therefore

$$
k^{-2}=\frac{s^{2}}{16}-2 \pi^{2} c_{1}^{2}=\frac{s^{2}}{16}-2 \pi^{2}(2 \chi+3 \sigma)=\frac{s^{2}}{16}+16 \pi^{2}(\mathbf{g}-1) .
$$

The traceless part of the Ricci tensor of $\tilde{g}$ can now be found as follows:

$$
\begin{aligned}
\tilde{r}_{0}(X, Y) & =\tilde{\rho}_{0}(X, \tilde{J} Y)=k^{-1} \omega(X, \tilde{J} Y)=k^{-1} g(J X, \tilde{J} Y) \\
& =k^{-1} u^{-2} \tilde{g}(J X, \tilde{J} Y)=-k^{-1} u^{-2} \tilde{\omega}(J X, Y) \\
& =-u^{-2} \rho_{0}(J X, Y)=u^{-2} r_{0}(X, Y) .
\end{aligned}
$$

On the other hand, since $\tilde{g}=u^{2} g$, we have from ([5], (1.161b)) that

$$
\tilde{r}_{0}=r_{0}+2 u\left(\nabla d\left(u^{-1}\right)+\frac{\Delta\left(u^{-1}\right)}{4} g\right),
$$

and hence from the above calculation

$$
u^{-2} r_{0}=r_{0}+2 u\left(\nabla d\left(u^{-1}\right)+\frac{\Delta\left(u^{-1}\right)}{4} g\right) .
$$

Using equation (7) we find that

$$
\Delta\left(u^{-1}\right)=-2 u^{-3}|d u|^{2}-u^{-2} \Delta u=-2 u^{-3}|d u|^{2}-\frac{s\left(u-u^{-1}\right)}{6},
$$

and therefore

$$
\nabla d\left(u^{-1}\right)=\frac{\left(u^{-3}-u^{-1}\right)}{2} r_{0}+\left(\frac{u^{-3}|d u|^{2}}{2}+\frac{s\left(u-u^{-1}\right)}{24}\right) g .
$$

In particular, at a maximum of $u^{-1}$ the Hessian of $u^{-1}$ is given by

$$
\nabla d\left(u^{-1}\right)=\frac{\left(u-u^{-1}\right)}{2}\left(\frac{s}{12} g-u^{-2} r_{0}\right) .
$$

Let $p \in M$ be any point on our manifold. Since $r_{0}$ is a traceless symmetric tensor of type $(1,1)$, we can find an orthonormal base $\left\{e_{1}, J e_{1}, e_{2}, J e_{2}\right\}$ of $T_{p} M$ such that $r_{0}$ can be represented by the matrix

$$
\left(\begin{array}{rrrr}
\lambda & 0 & 0 & 0 \\
0 & \lambda & 0 & 0 \\
0 & 0 & -\lambda & 0 \\
0 & 0 & 0 & -\lambda
\end{array}\right),
$$


where $\lambda$ is the positive constant given by

$$
\lambda=\frac{\left|r_{0}\right|}{2}=\frac{\left|\rho_{0}\right|}{\sqrt{2}}=\frac{|\tilde{\omega}|}{|k| \sqrt{2}}=\frac{u^{2}}{|k|} .
$$

The tensor $\left(\frac{s}{12} g-u^{-2} r_{o}\right)$ can now be represented by the matrix

$$
\left(\begin{array}{cccc}
\frac{s}{12}-\frac{1}{|k|} & 0 & 0 & 0 \\
0 & \frac{s}{12}-\frac{1}{|k|} & 0 & 0 \\
0 & 0 & \frac{s}{12}+\frac{1}{|k|} & 0 \\
0 & 0 & 0 & \frac{s}{12}+\frac{1}{|k|}
\end{array}\right)
$$

and since

$$
\frac{s}{12}-\frac{1}{|k|}=\frac{s}{12}-\sqrt{\frac{s^{2}}{16}+16 \pi^{2}(\mathbf{g}-1)} \leq \frac{s}{12}-\sqrt{\frac{s^{2}}{16}}=-\frac{s}{6}<0,
$$

the tensor is never semi-definite. But at the maximum of $u^{-1}$ the Hessian must be negative semi-definite, and hence from equation (9) we have that $u^{-1}=u=1$ at the maximum of $u^{-1}$, and by equation (6) we conclude that $u=1$ and hence $\tilde{g}=g$ everywhere.

Now the Ricci form satisfies

$$
\nabla \rho=\frac{s}{4} \nabla \omega+\nabla \rho_{0}=0 .
$$

Thus $g$ is generalized Kähler-Einstein. Since $\tilde{g}=g$ (or since $g$ is generalized Kähler-Einstein with non-vanishing $\rho_{0}$ ), we have that $g$ is Kähler with respect to two complex structures $J$ and $\tilde{J}$ inducing opposite orientations. Therefore the holomony [5] is a subgroup of $U(1) \times U(1)$ and the universal cover $(\hat{M}, \hat{g})$ of $(M, g)$ must be a Riemannian product $(\hat{M}, \hat{g})=\left(M_{1}, g_{1}\right) \times\left(M_{2}, g_{2}\right)$ of a pair of complete simply connected surfaces. Clearly the scalar curvature of each $\left(M_{i}, g_{i}\right)$ must be constant and since $s>0$ (but also for topological reasons [12]), we must have that at least one of the surfaces is a two sphere. Thus $(\hat{M}, \hat{g})=S^{2} \times\left(M_{2}, g_{2}\right)$. Since the genus of $\Sigma$ is at least one, $\left(M_{2}, g_{2}\right)$ must be either $\mathrm{C}$ or $\mathrm{CH}_{1}$ with their canonical metric. The rest of the proof follows along the same line of reasoning as in the proof of ([12], Theorem 4). In order to make this paper reasonably self-contained we repeat the arguments here. The holomony of $(\hat{M}, \hat{g})$ is $U(1) \times U(1)$ so the lift of $J$ on $M$ must coincide with the product complex structure, once the factors are correctly oriented. Since 
the genus of $\Sigma$ is at least one, we have that $\pi_{1}(M)=\pi_{1}(\Sigma)$ is non-trivial and acts on $S^{2} \times\left(M_{2}, g_{2}\right)$ by holomorphic isometries sending any compact holomorphic curve $S^{2} \times\{p t\}$ to another curve of this form. The induced action on $M_{2}$ is moreover free and proper, since $S^{2}$ is compact and every rotation of $S^{2}$ has a fixed point. Thus $M=\left(S^{2} \times M_{2}\right) / \pi_{1}(\Sigma)$ is biholomorphic to $\tilde{\Sigma} \times_{\rho} \mathrm{C} P_{1}$ for some compact Riemann surface $\tilde{\Sigma}$ and some representation $\rho: \pi_{1}(\tilde{\Sigma}) \rightarrow \mathrm{PSU}(2)=\mathrm{SO}(3)$. By uniqueness of ruling this biholomorphism must be a bundle biholomorphism inducing a biholomorphism between $\tilde{\Sigma}$ and $\Sigma$. Thus $M=\mathrm{P}(E) \rightarrow \Sigma$ is a flat $\mathrm{C} P_{1}$ bundle and $E$ is therefore quasi-stable.

AcKnowledgement. The author would like to thank C. LeBrun, J. E. Andersen, K. Akutagawa and H. Pedersen for very helpful conversations.

\section{REFERENCES}

1. Aubin, T., Nonlinear Analysis on Manifolds. Mong-Ampère Equations, Springer-Verlag 1982.

2. Barth, T., Peters, C. and Van de Ven, A., Compact Complex Surfaces, Springer, Berlin 1984.

3. de Bartolomeis, P., Burns, D., Stability of vector bundles and extremal metrics, Invent. Math 92 (1988), 403-407.

4. Beauville, A., Complex Algebraic Surfaces, Cambridge Univ. Press, Cambridge 1983.

5. Besse, A., Einstein Manifolds, Springer, Berlin 1987.

6. Calabi, E., Extremal Kähler Metrics, Sem. on Diff. Geom., Princeton Univ. Press (1982), 259-290.

7. Calabi, E., Extremal Kähler Metrics II, Diff. Geometry and Complex Analysis (ed. I. Chavel and H. M. Farkas) Springer-Verlag 1985.

8. Friedman, R., Morgan, J. W., Algebraic Surfaces and Seiberg-Witten Invariants, J. Algebraic Geom. 6 (1997), 445-479.

9. Hitchin, N. J., On Compact Four-Dimensional Einstein Manifolds, J. Differential Geom. 9 (1974), 435-442.

10. Kronheimer, P., Mrowka, T., The Genus of Embedded Surfaces in the Complex Projective Plane, Math. Res. Lett. 1 (1994), 797-808.

11. LeBrun, C., On the Scalar Curvature of Complex Surfaces, Geom. Funct. Anal. 5 (1995), 619-628.

12. LeBrun, C., Polarized 4-Manifolds, Extremal Kähler Metrics, and Seiberg-Witten Theory, Math. Res. Lett. 3 (1995), 653-662.

13. LeBrun, C., Yamabe Constants and the Perturbed Seiberg-Witten Equations, Comm. Anal. Geom. 5 (1997), 535-553.

14. Lee, J. and Parker, T., The Yamabe Problem, Bull. Amer. Math. Soc. 17 (1987), 37-91.

15. Li, T. J., Liu, A., General Wall Crossing Formula, Math. Res. Lett. 2 (1995), 797-810.

16. Matsushima, Y., Remarks on Kähler-Einstein metrics of positive scalar curvature, Nagoya Math. J. 46 (1972), 161-173.

17. Narasimhan, M. S., Seshadri, C. S., Stable and Unitary Vector Bundles on a Riemann surface, Ann. of Math. 82 (1965), 540-567.

18. Schoen, R., Conformal Deformation of a Riemannian Metric to Constant Scalar Curvature, J. Differential Geom. 20 (1984), 478-495.

19. Taubes, C.H., $S W \Rightarrow$ Gr: From the Seiberg-Witten Equations to Pseudo-Holomorphic Curves, J. Amer. Math. Soc. 9 (1996), 845-918. 
20. Thorpe, J. A., Some Remarks on the Gauss-Bonnet Formula, J. Appl. Math. Mech. 18 (1969), 779-786.

21. Witten, E., Monopoles and Four-Manifolds, Math. Res. Lett. 1 (1994), 769-796.

22. Yang, K., Complex Algebraic Geometry, An Introduction to Curves and Surfaces, Marcel Dekker, New York 1991.

DEPARTMENT OF MATHEMATICAL SCIENCES

UNIVERSITY OF AARHUS

BYGNING 530

8000 ÅRHUS C

DENMARK 